\section{Equity in Precision Medicine: Is It Within Our Reach?}

\author{
Katrina Armstrong, MD, MSCE
}

Disparities in health and healthcare in the United States are widespread, severe, and unacceptable. Advances in genomics and other precision medicine disciplines over the past several decades have created an important opportunity to reduce health disparities through the development of better diagnostic and predictive tools that reduce the impact of bias and stereotypes on clinical decision-making. ${ }^{1}$ However, this promise remains largely unrealized. In fact, lack of inclusion of diverse populations in precision medicine research and lack of access to precision medicine tools among disadvantaged populations risk creating the perfect storm wherein the greatest scientific advances of the past decades are on track to exacerbate rather than alleviate these disparities.

Evidence of disparities in the uptake of one form of precision medicine application, genomic tests, has been increasing for more than a decade. One of the first genomic tests to become clinically available was testing for mutations in the BRCA1 and BRCA2 genes, which hit the clinic more than 20 years ago. Perhaps not surprisingly, BRCA1/2 testing is also the first widely available genetic test to demonstrate large and persistent disparities in use. ${ }^{2}$ Multiple studies confirm that black or African American women are less likely to undergo BRCA1/2 testing than white women, even when they have the same risk of carrying a mutation, same insurance coverage, and same attitudes about the benefits and risks of testing. Furthermore, this disparity exists among women with a breast cancer diagnosis and those at risk based on family history alone-the 2 key populations who can benefit from BRCA1/2 testing.

However, this disparity in the use of genomic tests does not appear to be restricted to germline testing for hereditary cancer risk. More recently, use of somatic testing in breast tumors for gene expression patterns, marketed under the brand name Oncotype DX, has also been found to be lower among minority women. The article elsewhere in this issue, titled "Racial and Ethnic Disparities in Oncotype Dx Test Receipt in a Statewide Population-Based Study," adds further fuel to this fire. In a well-executed population-based study of women with newly diagnosed breast cancer, black and Hispanic women were substantially less likely to have undergone testing, among both those who met testing eligibility criteria based on tumor characteristics and those who did not meet testing eligibility criteria. Again, these disparities are substantial, with eligible non-Hispanic black and Hispanic women having two-thirds the odds of having undergone testing compared with non-Hispanic white women.

Despite the number of studies demonstrating disparities in uptake of precision medicine, our understanding of the factors driving those disparities remains limited. Although insurance coverage and patient affordability are critical determinants of access to any health service, large disparities in use of genomic tests have been demonstrated in insured populations. Furthermore, the contribution of lack of insurance may be restricted for somatic testing of tumor samples given that many uninsured patients become eligible for Medicaid coverage at the time of cancer diagnosis. Generosity of insurance coverage varies substantially across payors and plans, and more research is needed to know whether minority women have coverage that is less likely to include genomic tests or whether they face more administrative barriers to obtaining appropriate counseling or coverage for those tests.

Just as differences in insurance coverage do not appear to be the primary driver of these disparities to date, it has also proven difficult to explain disparities in genomic tests by differences in other patient characteristics. Test eligibility can vary across race

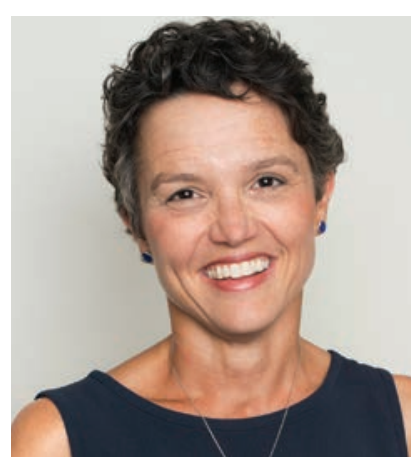

Katrina Armstrong, MD, MSCE

Katrina Armstrong, MD, MSCE, is the Jackson Professor at Harvard Medical School, and Physician-inChief, Department of Medicine, at Massachusetts General Hospital (MGH). Dr. Armstrong is a graduate of Johns Hopkins University School of Medicine. She was chief resident in Medicine at Johns Hopkins, and completed a general internal medicine fellowship and Masters of Science in Clinical Epidemiology at the University of Pennsylvania (Penn). In 1998, she joined the faculty at Penn and became Chief of the Division of General Internal Medicine. At Penn, she also served as Associate Director of the Abramson Cancer Center and Co-Director of the Robert Wood Johnson Clinical Scholars Program. In April 2013, she was appointed as physician-in-chief of the MGH Department of Medicine.

Dr. Armstrong is an internationally recognized investigator in the areas of medical decision-making, quality of care, and cancer prevention and outcomes. In addition to her career in health policy and disparities research, Dr. Armstrong is a dedicated practicing internist with a longitudinal primary care practice and rotations. Over her career, Dr. Armstrong has developed and led courses on clinical decisionmaking at Penn and MGH, and founded multiple innovative educational programs.

The ideas and viewpoints expressed in this commentary are those of the author and do not necessarily represent any policy, position, or program of NCCN. 
or ethnicity given differences in tumor characteristics or family history, but disparities exist even among women who clearly meet eligibility criteria. In addition, differences in sociodemographic factors, such as education or household income, explain a small proportion of a racial disparity in precision medicine in some studies. However, disparities universally persist after adjusting for these factors. Building on concerns about the history of eugenics and genetic discrimination, several studies have found significant levels of public concern about how their genomic information could be used by governmental or other organizations, with some studies demonstrating higher levels of concern among minority groups. ${ }^{3}$ However, the very limited research available to date that has measured racial differences in attitudes and in testing in the same population has generally found that the differences in attitudes explain very little of the testing disparity. ${ }^{2,4}$ Factors such as health literacy, social support, and trust that have been linked to disparities in healthcare in other settings are largely unstudied in precision medicine disparities and offer an important next step for research in this area.

Most recently, attempts to explain these disparities have returned their focus to the providers and systems in which the care is delivered. In a recent populationbased study of women with incident breast cancer, the disparity in use of BRCA1/2 testing between black and white women was substantially explained by differences in recommendations for testing by the patient's treating surgeon or oncologist. ${ }^{4}$ Another study found that differences in the site of care fully explained differences in use of Oncotype DX testing between black and white women. ${ }^{5}$ Why might healthcare providers be contributing to these disparities? Although issues of bias and stereotype are always important to consider in healthcare disparities, there are several reasons to believe that differences in healthcare provider behavior in this setting may be driven more by lack of awareness and uncertainty than by bias and stereotypes.

Until the past several years, genomic research has largely focused on populations of European ancestry. The underrepresentation of minorities is a widespread problem for clinical research in the United States but has been particularly significant in genomic research. Given that ancestry is correlated with genetic variation, concerns about confounding by population stratification led many investigators to exclude patients with non-European ancestry from both gene discovery and genetic association studies. Not surprisingly, the resultant relative lack of data in non-European ancestral groups means that early precision medicine tools risked being irrelevant or inaccurate for US minority populations.

This problem was further exacerbated by the low levels of use of clinical genomic tests among minority patients, thereby slowing the rate at which information was gathered through clinical practice. For example, initial studies of the prevalence of pathogenic and likely pathogenic mutations in BRCA1/2 included very few women of African ancestry, perhaps contributing to the initial perceptions that pathogenic mutations were less common among African American women than among white women in the United States. Furthermore, because very few African American women underwent clinical or research testing, a relatively high proportion of the variants discovered in the women who did undergo testing did not have sufficient population data to be able to categorize them as pathogenic or nonpathogenic. The rate of variants of unknown significance in BRCA1/2 test results among African American women has declined substantially over the past 10 years, but it is not clear whether the medical community has kept up with that change. Similarly, it wasn't until the recent creation of the Exome Aggregation Consortium (ExAC) database that reference genomic data became available for many ancestral groups, although Middle Eastern and some African populations remain underrepresented. ${ }^{6}$

Although the clinical utility of many different genomic tests continues to be debated, little doubt exists that the delay in collecting data on genomic variation 
in minority populations has created additional uncertainty about the utility of these tests in minority patients. One relatively recent study found that more than a third of physicians thought that genomic medicine applications were only applicable to certain racial or ethnic subpopulations. ${ }^{7}$ Greater uncertainty about the applicability of these tests to minority patients results in physicians being less likely to recommend the tests to their patients. ${ }^{8}$ Similarly, uncertainty about the relevance of these tests is likely to contribute to the persistent lower levels of awareness of genetic testing among minority populations in the United States. ${ }^{9}$ These low levels of awareness will reduce patient requests for testing, further dampening the rates of testing among eligible minority patients.

Major advances in our understanding of human biology have created a new paradigm for medical care, now termed precision medicine. ${ }^{10}$ Precision medicine seeks to use a broad array of information on individual variation to improve prevention, diagnosis, and treatment across medicine. Given the rapidity at which these efforts are moving forward, it is critical to ensure that uncertainty about the applicability of these new tools to minority populations does not further widen already unacceptable disparities in healthcare in the United States. Recent advances, such as the ExAC database and the commitment to funding sites with enhanced diversity in the National Human Genome Research Institute's genomic medicine portfolio, are important steps forward, making it clear that achieving equity in precision medicine is both challenging and within our reach.

\section{References}

1. Armstrong K. Genomics and health care disparities: the role of statistical discrimination. JAMA 2012;308:19791980.

2. Armstrong K, Micco E, Carney A, et al. Racial differences in the use of BRCA1/2 testing among women with a family history of breast or ovarian cancer. JAMA 2005;293:1729-1736.

3. Zimmerman RK, Tabbarah M, Nowalk MP, et al. Racial differences in beliefs about genetic screening among patients at inner-city neighborhood health centers. J Natl Med Assoc 2006;98:370-377.

4. McCarthy AM, Bristol M, Domchek SM, et al. Health care segregation, physician recommendation, and racial disparities in BRCA1/2 testing among women with breast cancer. J Clin Oncol 2016;34:2610-2618.

5. Guth AA, Fineberg S, Fei K, et al. Utilization of Oncotype DX in an inner city population: race or place? Int J Breast Cancer 2013;2013:653805.

6. Lek M, Karczewski KJ, Minikel EV, et al. Analysis of protein-coding genetic variation in 60,706 humans. Nature 2016;536:285-291.

7. Petersen KE, Prows CA, Martin LJ, Maglo KN. Personalized medicine, availability, and group disparity: an inquiry into how physicians perceive and rate the elements and barriers of personalized medicine. Public Health Genomics 2014;17:209-220.

8. Unequal Treatment: Confronting Racial and Ethnic Disparities in Healthcare. Washington, DC: The National Academies Press; 2002.

9. Pagan JA, Su D, Li L, et al. Racial and ethnic disparities in awareness of genetic testing for cancer risk. Am J Prev Med 2009;37:524-530.

10. Ashley EA. The precision medicine initiative: a new national effort. JAMA 2015;313:2119-2120. 